\title{
Intestinal Dysbiosis Is Associated with Altered Short-Chain Fatty Acids and Serum-Free Fatty Acids in Systemic Lupus Erythematosus
}

\author{
Javier Rodríguez-Carrio', Patricia López², Borja Sánchez', Sonia González³, \\ Miguel Gueimonde ${ }^{1}$, Abelardo Margolles ${ }^{1 *}$, Clara G. de los Reyes-Gavilán ${ }^{1 *}$ and \\ Ana Suárez ${ }^{2}$
}

'Department of Microbiology and Biochemistry of Dairy Products, Instituto de Productos Lácteos de Asturias (IPLA-CSIC), Villaviciosa, Asturias, Spain, ${ }^{2}$ Area of Immunology, Department of Functional Biology, University of Oviedo, Oviedo, Asturias, Spain, ${ }^{3}$ Area of Physiology, Department of Functional Biology, University of Oviedo, Oviedo, Asturias, Spain

OPEN ACCESS

Edited by:

lan Marriott,

University of North Carolina at

Charlotte, USA

Reviewed by:

Martin Rumbo,

National University of La Plata

(CONICET)

Paola Gauggin Cano,

Centro de Referencia Para

Lactobacilos (CONICET)

${ }^{*}$ Correspondence:

Abelardo Margolles

amargolles@ipla.csic.es;

Clara G. de los Reyes-Gavilán greyes_gavilan@ipla.csic.es

Specialty section:

This article was submitted to Microbial Immunology,

a section of the journal

Frontiers in Immunology

Received: 04 October 2016 Accepted: 05 January 2017

Published: 23 January 2017

Citation:

Rodríguez-Carrio J, López P,

Sánchez B, González S,

Gueimonde $M$, Margolles A,

de los Reyes-Gavilán CG and

Suárez A (2017) Intestinal Dysbiosis

Is Associated with Altered

Short-Chain Fatty Acids and

Serum-Free Fatty Acids in Systemic

Lupus Erythematosus.

Front. Immunol. 8:23.

doi: 10.3389/fimmu.2017.00023
Metabolic impairments are a frequent hallmark of systemic lupus erythematosus (SLE). Increased serum levels of free fatty acids (FFA) are commonly found in these patients, although the underlying causes remain elusive. Recently, it has been suggested that factors other than inflammation or clinical features may be involved. The gut microbiota is known to influence the host metabolism, the production of short-chain fatty acids (SCFA) playing a potential role. Taking into account that lupus patients exhibit an intestinal dysbiosis, we wondered whether altered FFA levels may be associated with the intestinal microbial composition in lupus patients. To this aim, total and specific serum FFA levels, fecal SCFA levels, and gut microbiota composition were determined in 21 SLE patients and 25 healthy individuals. The Firmicutes to Bacteroidetes (F/B) ratio was strongly associated with serum FFA levels in healthy controls $(\mathrm{HC})$, even after controlling for confounders. However, this association was not found in lupus patients, where a decreased F/B ratio and increased FFA serum levels were noted. An altered production of SCFA was related to the intestinal dysbiosis in lupus, while SCFA levels paralleled those of serum FFA in HC. Although a different serum FFA profile was not found in SLE, specific FFA showed distinct patterns on a principal component analysis. Immunomodulatory omega-3 FFA were positively correlated to the F/B ratio in $\mathrm{HC}$, but not in SLE. Furthermore, divergent associations were observed for pro- and anti-inflammatory FFA with endothelial activation biomarkers in lupus patients. Overall, these findings support a link between the gut microbial ecology and the host metabolism in the pathological framework of SLE. A potential link between intestinal dysbiosis and surrogate markers of endothelial activation in lupus patients is supported, FFA species having a pivotal role.

Keywords: free fatty acids, systemic lupus erythematosus, dysbiosis, microbiota, short-chain fatty acids

\section{INTRODUCTION}

Epidemiological studies have consistently shown an increase in the prevalence and severity of a number of metabolic disorders in patients with systemic lupus erythematosus (SLE) compared to the general population (1-3). Among them, metabolic syndrome, disturbed glucose metabolism, or altered lipid metabolism are the most relevant. These disorders are related to an increased risk of 
cardiovascular disease (CVD) development, the most important cause of mortality in $\operatorname{SLE}(4,5)$, thus highlighting the clinical relevance of the metabolic alterations in SLE.

Immune dysregulation and chronic inflammation are known to promote endothelial dysfunction in $\operatorname{SLE}(6,7)$. Increased levels of pro-inflammatory cytokines [such as tumor necrosis factor alpha (TNF $\alpha$ ), interleukin-8 (IL-8), and monocyte chemoattractant protein-1 (MCP-1)], adipokines, and autoantibodies are associated with the progression of endothelial dysfunction toward atherosclerosis development (6). At the local level, these mediators can impair the balance between endothelial repair and damage, whereas a number of systemic effects can be also triggered, including a shift to a pro-oxidant status (8) and altered lipid metabolism. In this scenario, although the underlying mechanisms are not totally understood, the relationship between systemic inflammation, metabolic disorders, and CVD may be explained, at least in part, by the free fatty acids (FFA) (9). FFA are fatty acid molecules released from adipocytes and several cell types upon lipolysis (10). Increased FFA levels in serum have been described in several metabolic disorders. Moreover, elevated serum FFA have also been found in immune-mediated diseases, such as SLE or rheumatoid arthritis, although striking differences were noted between both conditions (11). Rather than inflammatory or clinical parameters, the body mass index (BMI) was found to be the main predictor of FFA serum levels in lupus (11). However, these clinical studies did not allow the elucidation of the exact mediators and mechanisms involved.

Obesity is the result of an imbalance between energy intake and expenditure, which results in an excess of fat accumulation. However, several epidemiological studies have identified people with low BMI exhibiting markers of metabolic dysfunction (12, 13). Similarly, healthy metabolic profiles are found in a subset of obese subjects $(14,15)$, hence suggesting that metabolic dysfunction (that is, impaired fatty acid mobilization) rather than adiposity should be considered as the underlying cause. Therefore, it is feasible that factors related to energy intake and expenditure may underlie altered FFA levels and thus, metabolic disorders.

A mounting body of evidence shows that the gut microbiota can influence the host metabolism as well as the energy harvest and storage (16-18). Actually, the gut microbiota is seen by some authors as a separate endocrine organ involved, through a molecular cross talk with the host, in the maintenance of energy homeostasis and fat deposition (19). Currently, extensive research efforts have been focused on deciphering the basis of the cross talk between the microbiota and the host metabolism in the development and progression of host diseases and have revealed the relevance of the intestinal microbiota-host metabolism axis mediated by different bacterial and host metabolites $(20,21)$. Thus, it may be speculated that changes in the intestinal microbial ecology could disrupt this homeostatic cross talk and precipitate the development of pathological outcomes in the host.

Recently, we have reported that SLE patients exhibit an altered intestinal composition compared to healthy subjects, mainly characterized by a decreased abundance of members of the Firmicutes phylum and an overrepresentation of those of Bacteroidetes (22). However, the clinical impact of this
SLE-associated intestinal dysbiosis remains to be elucidated. Taking into account the former assumptions, we hypothesized that altered gut microbiota composition in SLE may underlie increased FFA serum levels. Accordingly, the main aims of the present report were (i) to analyze the potential association between the microbiota composition and FFA serum levels, (ii) to elucidate whether microbial metabolites can have a role in this interaction, (iii) to evaluate whether a different profile of FFA can be found in lupus patients, and (iv) to study the associations of these parameters with clinically relevant serum biomarkers.

\section{MATERIALS AND METHODS}

\section{Ethical Approval}

Ethical approval for this study was obtained from the Institutional Review Board (Comité de Ética de Investigación Clínica del Principado de Asturias) in compliance with the Declaration of Helsinki. All participants were informed and gave a signed informed consent prior their inclusion in the study.

\section{Patients and Controls}

Our study involved 21 SLE patients, all fulfilling classification criteria for SLE. According to the 1982 revised criteria from the American College of Rheumatology, a definitive SLE diagnosis can be established when a patient exhibit at least 4 out of the 11 SLE criteria (malar rash, discoid lesions, photosensitivity, oral ulcers, arthritis, serositis, renal disorders, neurological disorder, cytopenia, raised anti-DNA titers, and positivity to antinuclear antibodies) (23). A complete clinical examination, including Systemic Lupus Erythematosus Disease Activity Index (SLEDAI) calculation and anti-dsDNA autoantibodies assessment, was performed at the time of sampling. All patients were in remission (SLEDAI <8) at the sampling time. Information on clinical features along the disease course as well as the therapies received during the last 6 months was obtained from their clinical records. A group of 25 age- [median 43.50 (range 23.00-63.00) years] and gender-matched (23 females) healthy individuals recruited from the general population was included as the healthy controls (HC). Patients and controls did not differ in age $(p=0.293)$ and gender distribution $(p=0.495)$. Exclusion criteria were the history of recent infections, diagnosis of metabolic diseases, or the use of antibiotics, glucocorticoids, or monoclonal antibodies in the previous 6 months.

Upon acceptance of the individuals to participate in the study, a strict overnight fast (more than $8 \mathrm{~h}$ ) blood sample was obtained in tubes without anticoagulant. Serum was collected, divided into aliquots, and samples were stored at $-80^{\circ} \mathrm{C}$ until experimental procedures were performed. Additionally, basic serum blood lipid analyses were carried out on fresh samples at the time of sampling, by standardized procedures.

\section{Quantification of Total FFA Serum Levels}

Total FFA serum levels were analyzed by a colorimetric enzymatic assay using a commercial kit (NEFA kit half-microtest, Roche Life 
Sciences, Penzberg, Germany) following the instructions from the manufacturer. Final absorbance was measured at $546 \mathrm{~nm}$, and the detection limit was $0.2 \mathrm{mM}$.

\section{Assessment of Serum FFA Profiles}

Individual FFA were analyzed in serum samples following a methyl-tert-butylether-based extraction protocol (MTBE) as previously described (24), with minor modifications. Briefly, serum samples $(100 \mu \mathrm{l})$ were spiked with $5 \mu \mathrm{l}$ of internal standard (600 ppm heptadecanoic acid). Proteins were precipitated by the addition of $200 \mu \mathrm{l}$ methanol chromasolv grade (Sigma Aldrich, MO, USA). Organic phases were obtained by the addition of 1,200 $\mu \mathrm{l}$ MTBE chromasolv grade (Sigma) followed by an incubation in an ultrasound water bath at $15^{\circ} \mathrm{C}$ for $30 \mathrm{~min}$. Finally, organic phases were isolated by centrifugation at 5,000 rpm $\left(7 \mathrm{~min}, 15^{\circ} \mathrm{C}\right.$ ) after the addition of $200 \mu \mathrm{lmilliQ}$ water. The extraction protocol was repeated once with $100 \mu \mathrm{l} \mathrm{MetOH,} 500 \mu \mathrm{l}$ MTBE, and $100 \mu \mathrm{l}$ milliQ $\mathrm{H}_{2} \mathrm{O}$. Lipid extracts were dried in a miVac centrifugal evaporator (Genevac Ltd., UK) and redissolved in $100 \mu \mathrm{l}$ of water:acetonitrile (38:62).

The analyses of fatty acids in the samples were performed in a Dionex Ultimate 3000 HPLC system (Thermo Scientific, Bremen, Germany) equipped with a column Zorbax Eclipse Plus C18 $(50 \mathrm{~mm} \times 2.1 \mathrm{~mm}, 1.8 \mu \mathrm{m})$. Mobile phases A and B were water and acetonitrile, respectively, both containing $0.1 \%$ of formic acid. Fatty acids were separated in an injection volume of $2 \mu \mathrm{l}$ by a gradient program as follows: $62 \%$ B (held for $4.5 \mathrm{~min}$ ) followed by a linear increase up to $100 \% \mathrm{~B}$ in $10 \mathrm{~min}$ (held for $1 \mathrm{~min}$ ). The column temperature was set at $45^{\circ} \mathrm{C}$. Mass detection was carried out in a Bruker Impact II q-ToF mass spectrometer with electrospray ionization, operating in the negative mode. The settings of the mass spectrometer were as follows: spray voltage $4.5 \mathrm{kV}$; drying gas flow $12 \mathrm{l} / \mathrm{min}$; drying gas temperature $250^{\circ} \mathrm{C}$; and nebulizer pressure 44 psi.

For quantification, calibration curves for each compound were prepared by dissolution of the pure standards in methanol to adequately encompass the expected concentration of the analytes in the samples. The calibration ranges were as follows: $0.4-12.5 \mu \mathrm{g} / \mathrm{ml}$ for EPA and $\gamma$-linolenic; $1.2-37.5 \mu \mathrm{g} / \mathrm{ml}$ for DHA and linolenic; $2.3-75 \mu \mathrm{g} / \mathrm{ml}$ for AA and palmitoleic; 3.9-125 $\mu \mathrm{g} / \mathrm{ml}$ for linoleic; and 7.8-250 $\mu \mathrm{g} / \mathrm{ml}$ for oleic, palmitic, and stearic acids. A good linearity was observed in all cases $\left(r^{2}>0.994\right)$. Heptadecanoic acid was used as internal standard to account for potential biases during the extraction protocol.

\section{Analysis of Fecal Microbiota}

Fecal sample collection and metagenomic analyses of fecal microbiota were performed as previously reported (22). Briefly, fresh fecal material was processed within $3 \mathrm{~h}$ from collection and immediately homogenized and stored at $-80^{\circ} \mathrm{C}$. Fecal DNA was extracted with a QIAampDNA stool minikit (Qiagen, Strasse, Germany). Then, 16S rRNA gene sequences were amplified, and 16S rRNA and gene-based amplicons were sequenced by an Ion Torrent PGM sequencing platform as described elsewhere (22).

\section{Analysis of Short-Chain Fatty Acids (SCFA) in Fecal Samples}

Analysis of SCFA (acetate, propionate, and butyrate) was performed by gas chromatography. Briefly, $1 \mathrm{~g}$ of fecal samples was diluted 1:10 in sterile PBS and homogenized in a LabBlender 400 stomacher (Seward Medical, London, UK) at full speed for $4 \mathrm{~min}$. Then, supernatants were obtained by centrifugation $(10.000 \times g$, $30 \mathrm{~min}, 4^{\circ} \mathrm{C}$ ), filtered through $0.2-\mu \mathrm{m}$ filters, mixed with $1: 10$ of ethyl butyric acid $(2 \mathrm{mg} / \mathrm{ml})$ as an internal standard, and stored at $-80^{\circ} \mathrm{C}$ until analysis.

A gas chromatograph 6890N (Agilent Technologies Inc., Palo Alto, CA, USA) connected to a mass spectrometry (MS) $5973 \mathrm{~N}$ detector (Agilent Technologies) and to a flame ionization detector was used for identification and quantification of SCFA. Data were collected using the Enhanced ChemStation G1701DA software (Agilent). Samples $(1 \mu \mathrm{l})$ were injected into the gas chromatograph equipped with an HP-Innowax capillary column (60-m length by $0.25-\mathrm{mm}$ internal diameter, with a $0.25-\mu \mathrm{m}$ film thickness; Agilent) using $\mathrm{He}$ as a gas carrier (flow rate of $1.5 \mathrm{ml} / \mathrm{min}$ ). The temperature of the injector was kept at $220^{\circ} \mathrm{C}$, and the split ratio was 50:1. Chromatographic conditions were as follows: initial oven temperature of $120^{\circ} \mathrm{C}, 5^{\circ} \mathrm{C} / \mathrm{min}$ up to $180^{\circ} \mathrm{C}, 1 \mathrm{~min}$ at $180^{\circ} \mathrm{C}$, and a ramp of $20^{\circ} \mathrm{C} / \mathrm{min}$ up to $220^{\circ} \mathrm{C}$ to clean the column. In the MS detector, the electron impact energy was set at $70 \mathrm{eV}$. The data collected were in the range of 25 to 250 atomic mass units (at 3.25 scans/s).

SCFA were identified by comparison of their mass spectra with those held in the HP-Wiley 138 library (Agilent) and by comparison of their retention times with those of the corresponding standards (Sigma Aldrich, St. Louis, MO, USA). The peaks were quantified as relative abundances with respect to the internal standard. The concentration (in millimolar) of each SCFA was calculated using the linear regression equations $\left(R^{2} \geq 0.99\right)$ from the corresponding standard curves.

\section{Analysis of Serum Biomarkers Soluble Biomarkers}

Serum levels of vascular endothelial growth factor (VEGF), granulocyte monocyte colony-stimulating factor (GM-CSF), and IL- 8 were analyzed by Cytometric Bead Arrays (BD Biosciences, NJ, USA) using a BD FACS Canto II and FACS Diva software.

Detection limits were $4.5,0.2$, and $1.2 \mathrm{pg} / \mathrm{ml}$, respectively.

Epidermal growth factor (EGF), TNF $\alpha, \mathrm{MCP}-1$, interferon gamma-inducible protein-10 (IP-10), and leptin serum levels were assessed by plate immunoassays using commercial kits by Peprotech (NJ, USA), following manufacturer's instructions. Detection limits were $3.9,3.9,8,3.9$, and $24 \mathrm{pg} / \mathrm{ml}$, respectively.

\section{Malondialdehyde (MDA)}

Malondialdehyde serum levels were determined by means of a colorimetric method using a commercial kit (LPO-596, Byoxytech, Oxis International, France). Final absorbance was read at $586 \mathrm{~nm}$. 


\section{Anthropometric Measures}

Height was measured using a stadiometer with an accuracy of $\pm 1 \mathrm{~mm}$ (Año-Sayol, Barcelona, Spain). The subjects stood barefoot, in an upright position and with the head positioned in the Frankfort horizontal plane. Weight was measured on a scale with an accuracy of $\pm 100 \mathrm{~g}$ (Seca, Hamburg, Germany).

\section{Nutritional Assessments}

Dietary intakes were assessed by means of an annual semiquantitative validated food frequency questionnaire including 160 items (25). During an interview by trained dietitians, subjects were asked, item by item, whether they usually ate each food and, if so, how much they usually ate. For this purpose, three different serving sizes of each cooked food were presented in pictures to the participants so that they could choose from up to seven serving sizes (from "less than the small one" to "more than the large one"). For some of the foods consumed, amounts were recorded in household units, by volume, or by measuring with a ruler. Information on the cooking practices, number and amount of ingredients used in each recipe, and other relevant information for the study was collected. Methodological issues concerning dietary assessment have been detailed elsewhere (25). The consumption of foods was converted into energy intake (kilocalories per day), macronutrients (carbohydrates, lipids, and proteins, grams per day), and total fiber (grams per day) using the nutrient food composition tables developed by the Centro de Enseñanza Superior de Nutrición Humana y Dietética (CESNID) (26). CESNID, a foundation, involves different institutions, universities, and companies related to the food and nutrition area, and its food composition databases are supported by the Spanish Association of Nutrition and Dietetics.

\section{Statistical Analyses}

Continuous variables were expressed as median (interquartile range) or mean \pm SD. Mann-Whitney $U$, Student's $t$ or KruskalWallis tests were performed to assess statistical differences. Correlations were analyzed by Spearman's rank or Pearson tests, depending on the distribution of the data. Categorical variables were summarized as $n(\%)$, and differences were analyzed by $\chi^{2}$ tests. A principal component analysis (PCA) was performed to avoid potential bias due to collinearity. The adequacy of the data was studied by Kaiser-Meyer-Olkin test and Bartlett test of sphericity. The number of components retained was based on eigenvalues $(>1)$, and loadings greater than 0.5 were used to identify the variables comprising each component. Unsupervised cluster analysis was performed based on squared Euclidean distances, and Ward's Minimum Variance method was used to identify the clusters. Heatmaps were generated under R package heatmap.2. SPSS 21.0, R 3.0.3, and GraphPad Prism 5.0 for Windows were used.

\section{RESULTS}

\section{Total FFA Serum Levels in SLE Patients: Association with Intestinal Dysbiosis}

The concentration of total FFA was measured in serum samples from 21 SLE patients and 25 matched HC (Table 1). SLE patients exhibited higher FFA serum levels (Table 1). Differences between groups in the levels of FFA remained significant after adjusting for age and gender $(p=0.024)$. Moreover, no associations were found with demographical parameters, cholesterol and triglycerides levels, and dietary intakes (Table 2). Furthermore, FFA were neither related to clinical manifestations (Table 3) (all $p>0.050)$ nor disease activity $(r=-0.349, p=0.169)$, duration $(r=-0.005$,

TABLE 1 | Serum-free fatty acids (FFA) levels, nutritional parameters and blood lipid profiles of the healthy controls $(\mathrm{HC})$ and systemic lupus erythematosus (SLE) patients recruited in this study.

\begin{tabular}{lccc}
\hline & HC $(\boldsymbol{n}=\mathbf{2 5})$ & SLE $(\boldsymbol{n}=\mathbf{2 1})$ & $\boldsymbol{p}$ \\
\hline FFA assessment & & & \\
Total FFA (mM) & $0.27(0.17)$ & $0.41(0.26)$ & 0.045 \\
& & & \\
Blood lipid analyses & & & \\
Total cholesterol (mg/dl) & $191.50(49.00)$ & $200.00(61.25)$ & 0.732 \\
HDL-cholesterol (mg/dl) & $62.00(14.75)$ & $62.00(24.00)$ & 0.740 \\
LDL-cholesterol (mg/dl) & $114.50(50.00)$ & $111.50(57.50)$ & 0.530 \\
Triglycerides (mg/dl) & $68.50(54.25)$ & $71.55(45.25)$ & 0.715 \\
Nutritional parameters & & & \\
Total energy (kcal/day) & $1,888.88(226.53)$ & $2,186.11(208.16)$ & 0.069 \\
Carbohydrates (g/day) & $202.50(51.60)$ & $205.46(102.95)$ & 0.944 \\
Lipids (g/day) & $78.13(29.32)$ & $79.42(63.57)$ & 0.789 \\
Proteins (g/day) & $96.98(15.81)$ & $102.60(34.30)$ & 0.782 \\
Fiber (g/day) & $24.68(6.17)$ & $26.47(6.57)$ & 0.609 \\
Body mass index (kg/m²) & $24.96(4.47)$ & $24.58(7.78)$ & 0.715 \\
\hline
\end{tabular}

Variables are represented as median (interquartile range) or $n$ (\%) unless otherwise stated. Differences in demographical and blood lipid variables were assessed by Mann-Withney $U$ tests, whereas differences in daily intakes were analyzed by multivariate analyses adjusted for confounders. Energy was adjusted by gender and age, whereas the rest of the nutrients were adjusted by gender, age, and energy.

TABLE 2 | Analysis of the correlation between serum FFA levels and demographical and nutritional features in healthy controls (HC) and systemic lupus erythematous (SLE) patients.

\begin{tabular}{|c|c|c|}
\hline & $\mathrm{HC}$ & SLE \\
\hline \multirow[t]{2}{*}{ Age } & $r=-0.260$ & $r=-0.179$ \\
\hline & $p=0.231$ & $p=0.451$ \\
\hline \multirow[t]{2}{*}{ Total cholesterol } & $r=-0.108$ & $r=-0.203$ \\
\hline & $p=0.625$ & $p=0.390$ \\
\hline \multirow[t]{2}{*}{ HDL-cholesterol } & $r=0.220$ & $r=0.114$ \\
\hline & $p=0.313$ & $p=0.633$ \\
\hline \multirow[t]{2}{*}{ LDL-cholesterol } & $r=-0.028$ & $r=-0.248$ \\
\hline & $p=0.898$ & $p=0.291$ \\
\hline \multirow[t]{2}{*}{ Triglycerides } & $r=-0.224$ & $r=-0.078$ \\
\hline & $p=0.305$ & $p=0.743$ \\
\hline \multirow[t]{2}{*}{$\mathrm{BMl}$} & $r=0.247$ & $r=0.214$ \\
\hline & $p=0.268$ & $p=0.366$ \\
\hline \multirow[t]{2}{*}{ Total energy } & $r=0.082$ & $r=-0.212$ \\
\hline & $p=0.710$ & $p=0.369$ \\
\hline \multirow[t]{2}{*}{ Carbohydrates } & $r=-0.107$ & $r=-0.311$ \\
\hline & $p=0.628$ & $p=0.182$ \\
\hline \multirow[t]{2}{*}{ Lipids } & $r=-0.048$ & $r=-0.220$ \\
\hline & $p=0.826$ & $p=0.352$ \\
\hline \multirow[t]{2}{*}{ Proteins } & $r=0.010$ & $r=-0.005$ \\
\hline & $p=0.964$ & $p=0.985$ \\
\hline \multirow[t]{2}{*}{ Fiber } & $r=0.125$ & $r=-0.394$ \\
\hline & $p=0.568$ & $p=0.086$ \\
\hline
\end{tabular}

Correlations were assessed by Spearman ranks tests ( $r$ coefficient and p-value is indicated for each parameter). 
TABLE 3 | Demographical and clinical parameters of the systemic lupus erythematosus (SLE) patients.

\begin{tabular}{lc}
\hline & SLE $(\boldsymbol{n}=\mathbf{2 1})$ \\
\hline Age, (years), mean (range) & $48.35(27.00-70.00)$ \\
Gender, (f/m) & $21 / 0$ \\
Age at diagnosis, (years) & $33.00(14.50)$ \\
Disease duration, (years), median (range) & $7.00(2.00-24.00)$ \\
SLEDAl score & $4.00(3.25)$ \\
Clinical manifestations, $\boldsymbol{n ( \% )}$ & \\
Malar rash & \\
Photosensitivity & $12(57.1)$ \\
Discoid lesions & $16(76.2)$ \\
Arthritis & $6(28.6)$ \\
Oral ulcers & $10(47.6)$ \\
Serositis & $10(47.6)$ \\
Renal disorder & $4(19.0)$ \\
Neurological disorder & $3(14.3)$ \\
Cytopenia & $0(0.0)$ \\
Autoantibodies, $\boldsymbol{n}(\%)$ & $11(52.4)$ \\
ANAs & \\
Anti-dsDNA titer, (U/ml), mean \pm SD & $21(100)$ \\
Anti-SSa & $25.30 \pm 33.89$ \\
Anti-SSb & $11(52.4)$ \\
Anti-Sm & $2(9.5)$ \\
Anti-RNP & $2(9.5)$ \\
Treatments, $\boldsymbol{n}$ (\%) & $1(4.8)$ \\
None or NSAIDs & \\
Antimalarials & $3(14.2)$ \\
\hline Variables are represent) \\
\\
\end{tabular}

Variables are represented as median (interquartile range) or n(\%), unless otherwise stated.

$p=0.982)$, or anti-dsDNA levels $(r=-0.350, p=0.130)$ in SLE. Therefore, parameters other than those indicated may explain the altered serum FFA levels registered in SLE.

Then, we wondered whether gut microbial composition may account for the increased FFA serum levels in SLE. To this aim, the associations between FFA levels and the main intestinal microbial groups analyzed by a metagenomic approach as already described (22) were assessed. As previously reported, diminished Firmicutes to Bacteroidetes (F/B) ratio characterized the intestinal dysbiosis found in SLE compared to healthy subjects [1.94 (1.51) vs 4.27 (5.93), $p<0.001$ ] (22). Among the phyla analyzed (Actinobacteria, Bacteroidetes, Firmicutes, Cyanobacteria, Euryarchaeota, Fusobacteria, Lentisphaerae, Proteobacteria, Tenericutes, TM7, Verrucomicrobia, and Synergistetes), FFA levels displayed opposite correlations with the Firmicutes and Bacteroidetes groups in $\mathrm{HC}$, but not in lupus patients (Table 4). Consequently, a negative association with the F/B ratio was observed in HC, but not in SLE (Figure 1). Moreover, this association remained significant after adjusting for potential confounders (Table 5). Therefore, F/B ratio was found to be the main predictor of FFA serum levels in HC, whereas this effect was not seen in lupus patients, hallmarked by a decreased $\mathrm{F} / \mathrm{B}$ ratio and elevated FFA levels.

Since some heterogeneity within groups in the FFA levels was observed and in order to gain more insight into the connections between gut microbiota and serum FFA, further analyses were performed. Focusing on the main microbial groups at the level
TABLE 4 | Association between serum-free fatty acids (FFA) levels and gut microbiota composition in healthy controls $(\mathrm{HC})$ and systemic lupus erythematosus patients (SLE).

\begin{tabular}{|c|c|c|}
\hline & $\mathrm{HC}$ & SLE) \\
\hline Actinobacteria & $\begin{array}{l}r=0.075 \\
p=0.733\end{array}$ & $\begin{array}{l}r=-0.415 \\
p=0.069\end{array}$ \\
\hline Bacteroidetes & $\begin{array}{c}r=0.721 \\
p<0.0001\end{array}$ & $\begin{array}{l}r=0.311 \\
p=0.182\end{array}$ \\
\hline Firmicutes & $\begin{array}{l}r=-0.574 \\
p=0.007\end{array}$ & $\begin{array}{l}r=-0.117 \\
p=0.622\end{array}$ \\
\hline Cyanobacteria & $\begin{array}{l}r=-0.120 \\
p=0.539\end{array}$ & $\begin{array}{l}r=-0.401 \\
p=0.080\end{array}$ \\
\hline Euryarchaeota & $\begin{array}{l}r=-0.213 \\
p=0.328\end{array}$ & $\begin{aligned} r & =-0.063 \\
p & =-0.792\end{aligned}$ \\
\hline Fusobacteria & $\begin{array}{l}r=-0.052 \\
p=0.812\end{array}$ & $\begin{array}{l}r=0.139 \\
p=0.560\end{array}$ \\
\hline Lentisphaerae & $\begin{array}{l}r=0.272 \\
p=0.210\end{array}$ & $\begin{array}{l}r=0.019 \\
p=0.937\end{array}$ \\
\hline Proteobacteria & $\begin{array}{l}r=-0.158 \\
p=0.471\end{array}$ & $\begin{array}{l}r=0.230 \\
p=0.329\end{array}$ \\
\hline Tenericutes & $\begin{array}{l}r=-0.105 \\
p=0.634\end{array}$ & $\begin{aligned} r & =-0.220 \\
p & =0.227\end{aligned}$ \\
\hline TM7 & $\begin{array}{l}r=0.015 \\
p=0.945\end{array}$ & $\begin{array}{l}r=0.018 \\
p=0.939\end{array}$ \\
\hline Verrucomicrobia & $\begin{array}{l}r=-0.325 \\
p=0.130\end{array}$ & $\begin{array}{l}r=0.209 \\
p=0.376\end{array}$ \\
\hline Synergistetes & $\begin{array}{l}r=-0.211 \\
p=0.334\end{array}$ & $\begin{array}{l}r=-0.104 \\
p=0.661\end{array}$ \\
\hline
\end{tabular}

The associations between serum FFA levels and the abundance of microbial groups at the level of phyla in HC and SLE patients were analyzed by Spearman ranks tests ( $r$ coefficient and $p$-value is indicated for each parameter). Statistical analyses with a p-value below 0.050 are highlighted in bold.

of phyla, individuals were classified into groups by means of a cluster analysis. Interestingly, two main clusters were identified (thereafter referred to as clusters I and II) (Figure 2A), mainly differing in the $\mathrm{F} / \mathrm{B}$ ratio $(8.84 \pm 5.53$ vs $1.70 \pm 0.76$, respectively; $p<0.0001)$. Notably, a distinct distribution of individuals was observed, as HC were mainly found within the cluster I (14/25), whereas SLE patients were marginally present in this group $(3 / 21$, $p=0.004)$. On the one hand, this result highlights a shift in the microbiota composition in SLE patients compared to HC, hence supporting an association between a biased distribution of the intestinal microbial groups and elevated FFA serum levels. More importantly, when FFA levels were compared among $\mathrm{HC}$ and SLE subjects stratified by microbial clusters, it was noted that HC grouping within the cluster II exhibited similar FFA serum levels as SLE patients (Figure 2B), thus reinforcing the relevance of the microbiota composition on the FFA serum levels.

Overall, our findings disclose a strong association between FFA levels in serum and specific groups of the gut microbiota in healthy individuals, but not in lupus patients where a profound intestinal dysbiosis was registered.

\section{SCFA and FFA Levels}

Our results point to a relationship between the gut microbiota and the host metabolism at the systemic level, but the actual mediators are unclear. Since SCFA may affect the human metabolism 

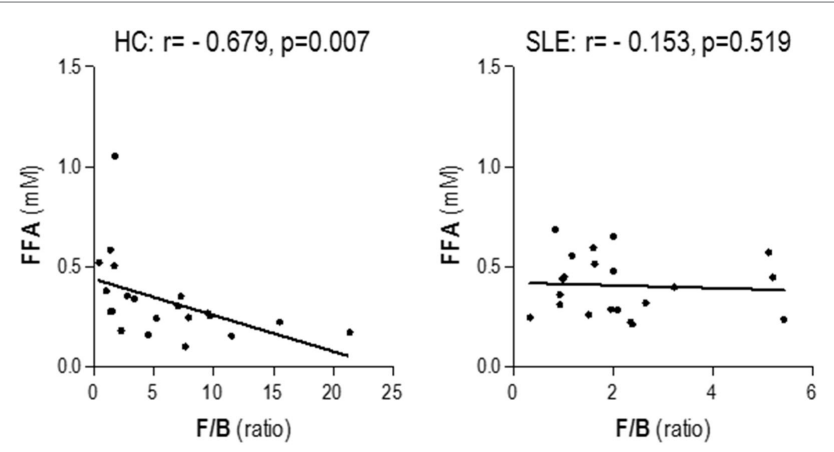

FIGURE 1 | Serum-free fatty acids (FFA) levels and Firmicutes to Bacteroidetes (F/B) ratio. The association between serum FFA levels and the F/B ratio was analyzed by Spearman's ranks correlation tests.

TABLE 5 | Firmicutes/Bacteroidetes (F/B) ratio is the main predictor of FFA levels in healthy controls (HC) but not in systemic lupus erythematosus (SLE) patients.

\begin{tabular}{llrcc}
\hline & & $\boldsymbol{\beta}$ & $\boldsymbol{B}[\mathbf{9 5 \%} \mathbf{C l}]$ & $\boldsymbol{p}$ \\
\hline HC & F/B ratio & -0.636 & $-0.334[-0.557,-0.111]$ & $\mathbf{0 . 0 0 7}$ \\
Age & -0.354 & $-0.652[-1.436,0.132]$ & 0.093 \\
Gender & 0.150 & $0.114[-0.179,0.407]$ & 0.405 \\
BMl & -0.039 & $-0.002[-0.022,0.018]$ & 0.832 \\
CRP & 0.191 & $0.133[-0.170,0.436]$ & 0.352 \\
Total energy & -0.010 & $0.001[-0.044,0.042]$ & 0.961 \\
Lipids & 0.276 & $0.003[-0.004,0.010]$ & 0.315 \\
Carbohydrates & -0.372 & $-0.002[-0.004,0.001]$ & 0.073 \\
Proteins & 0.092 & $0.001[-0.005,0.007]$ & 0.690 \\
Fiber & -0.223 & $-0.007[-0.020,0.005]$ & 0.239 \\
& & & \\
F/B ratio & -0.025 & $-0.014[-0.332,0.303]$ & 0.923 \\
Age & -0.229 & $-0.336[-1.315,0.643]$ & 0.466 \\
BME & 0.415 & $0.012[-0.008,0.032]$ & 0.205 \\
CRP & 0.186 & $0.072[-0.191,0.335]$ & 0.558 \\
& Total energy & -0.072 & $-0.007[-0.045,0.031]$ & 0.687 \\
Lipids & -0.193 & $-0.001[-0.005,0.003]$ & 0.698 \\
Carbohydrates & -0.265 & $-0.001[-0.003,0.002]$ & 0.649 \\
Proteins & 0.369 & $0.002[-0.002,0.006]$ & 0.308 \\
Fiber & -0.351 & $-0.006[-0.019,0.008]$ & 0.365
\end{tabular}

The association between F/B ratio and FFA serum levels in HC was studied by multiple lineal regression analysis including demographical parameters and nutritional intakes as potential confounders. HC: $R^{2}$ (model) $=0.762$; SLE: $R^{2}$ (model) $=0.426$. Statistical analyses with a p-value below 0.050 are highlighted in bold.

and an altered gut microbiota composition leads to a dysregulated SCFA production, the associations between fecal SCFA levels and those of serum FFA were analyzed.

On the one hand, higher levels of all SCFA studied were observed in lupus patients compared to HC (Table 6). However, no differences were found between SLE and HC when relative proportions were compared (all $p>0.050$ ). On the other hand, all SCFA exhibited a positive correlation with FFA serum levels in HC (Table 6).

Importantly, the $\mathrm{F} / \mathrm{B}$ ratio was negatively correlated to the fecal levels of propionate and butyrate in HC but not in SLE patients
(Figure 3). Notably, stronger associations were found in $\mathrm{HC}$ when only Bacteroidetes abundance was considered: propionate $(r=0.653, p<0.001)$ and butyrate $(r=0.623, p=0.002)$.

In order to gain further insight into the relevance of the intestinal microbiota and the SCFA production and serum FFA levels, we performed additional analyses by stratifying subjects according to the clusters obtained from the microbiota analysis (Figure 2) instead of their clinical condition. Interestingly, a negative association between F/B ratio and fecal SCFA was found in cluster I (propionate: $r=-0.621, p=0.024$ and butyrate: $r=-0.654$, $p=0.015$ ), but not in those grouped in cluster II (propionate: $r=-0.015, p=0.940$ and butyrate: $r=0.220, p=0.271$ ). Overall, this picture mirrors that of found for the $\mathrm{HC}$ vs SLE populations according to our previous findings and confirms a pivotal role of the intestinal microbiota in this scenario. However, SCFA and FFA levels did not remain associated after stratifying the whole population by the clusters, hence suggesting the involvement of additional factors, such as the clinical condition, to explain the connection between gut microbiota composition, SCFA, and serum FFA levels.

All these results highlight a role for the gut microbiota in the maintenance of serum FFA levels, SCFA having a potential role orchestrating this interaction. Additional factors, such as disease status, may also influence the outcome of the associations between gut microbiota composition and the interaction SCFA-FFA. Indeed, altered gut microbiota composition found in SLE patients was linked to an altered SCFA production and increased FFA levels in serum, thus supporting this hypothesis.

\section{FFA Profiling in SLE Patients}

Although elevated FFA levels were found in SLE, whether a global increase in all FFA species underlies this finding, or if some specific FFA were altered was not clear. To address this issue, a number of FFA species were measured, and the differences between SLE and $\mathrm{HC}$ were studied.

Overall, no striking differences were observed between patients and controls (Table 7). However, since some collinearity among FFA species existed, a PCA was carried out on the FFA species analyzed to avoid potential biases. PCA demonstrated a good adequacy of the data (KMO statistic $=0.781$, Barlett sphericity test $\left.p=10^{-44}\right)$, and three components were identified (eigenvalues $>1$ ) explaining $77.53 \%$ of the total variance. Based on their loadings, PC1 (53.32\% variance explained) retained $\gamma$-linolenic, palmitoleic, palmitic, oleic, linolenic, linoleic, and arachidonic acids, and PC2 (13.95\% variance explained) mainly retained EPA and DHA. Finally, PC3 (10.26\% variance explained) only retained stearic acid (Figure 4).

On the one hand, these results underlie the outstanding heterogeneity of FFA species, a clearly distinct pattern of grouping depending on their chemical structure (chain length or doublebond position) not being found. Globally, saturated, monounsaturated, and w6 fatty acids were grouped together within the PC1, whereas the most important anti-inflammatory w3 FFA did in the PC2. Stearic acid, of controversial immunological and metabolic role, was grouped in the third component. Hence, these findings support a functional, rather than structural, relationship of FFA. 


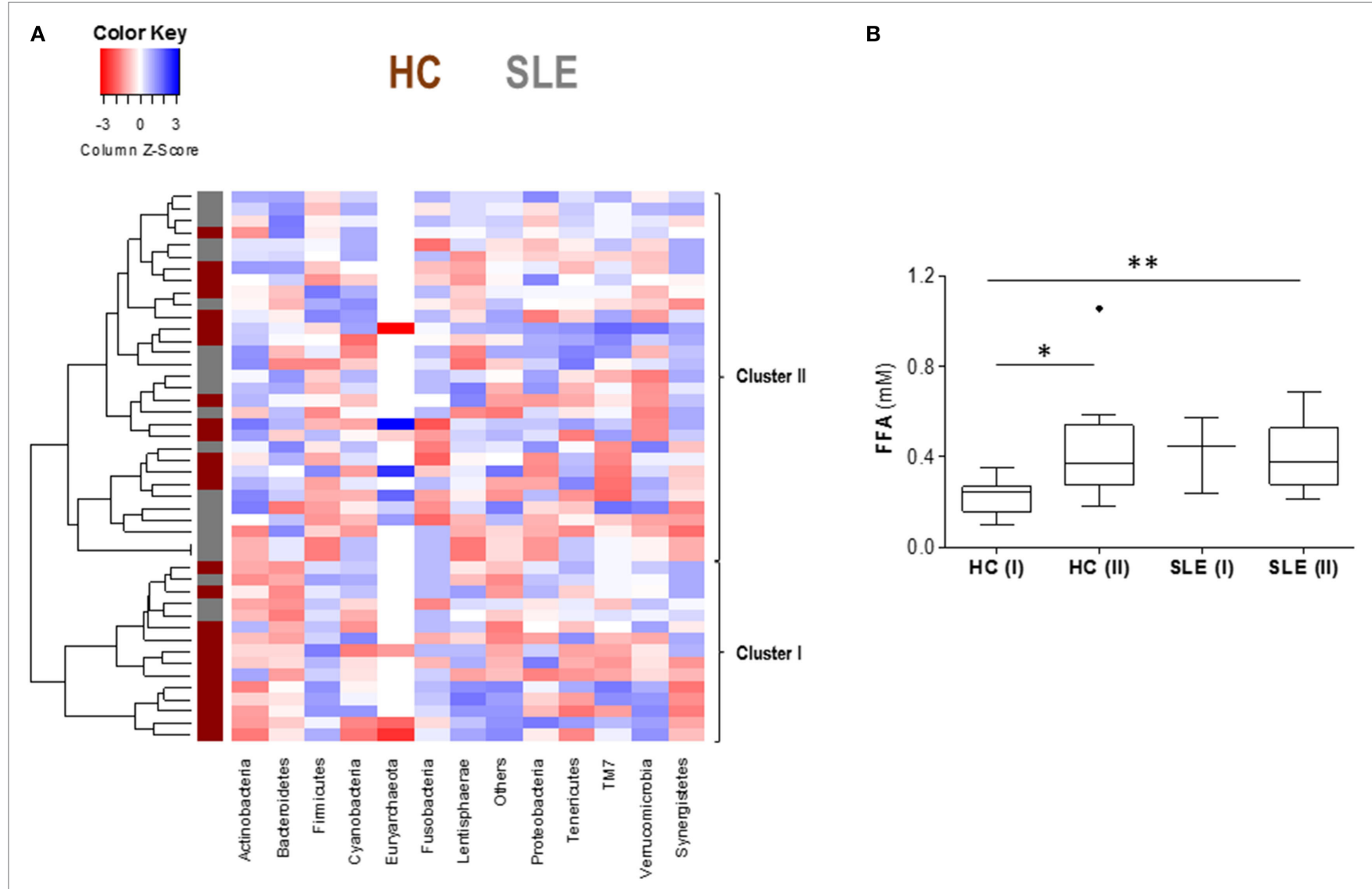

FIGURE 2 | Cluster analysis revealed a link between gut microbiota composition and total free fatty acids (FFA) serum levels. (A) Heatmap plotting gut microbiota composition, based on the main phyla, in healthy controls ( $\mathrm{HC}$ ) and systemic lupus erythematosus (SLE) patients. Tiles are colored based on the abundance of each group, red and blue colors indicating low or high levels, respectively. Each row represents an individual, and the vertical colored bar at the left represents HC (dark red) or SLE patient (gray). Two clusters were identified and are highlighted at the right (clusters I and II). (B) Comparison of total FFA serum levels among groups after stratifying by disease status (HC or SLE) and microbiota clusters (I or II). Boxes represent median and interquartile range, whereas whiskers represent minimum and maximum values. Differences were assessed by Kruskal-Wallis test and Dunn-Bonferroni post hoc correction for multiple comparisons tests. ${ }^{\star} p<0.050,{ }^{\star \star} p<0.010$

TABLE 6 | Analysis of fecal short-chain fatty acids (SCFA) levels and their correlation with serum-free fatty acids (FFA) levels in healthy controls (HC) and systemic lupus erythematosus (SLE) patients.

\begin{tabular}{lccc}
\hline & HC & SLE & $\boldsymbol{p}$ \\
\hline SCFA levels (mM) & & & \\
Acetate & $41.14(12.30)$ & $57.63(19.63)$ & $\mathbf{0 . 0 0 5}$ \\
Propionate & $11.96(8.72)$ & $20.61(9.80)$ & $\mathbf{0 . 0 0 3}$ \\
Butyrate & $7.78(3.97)$ & $10.50(7.14)$ & 0.075 \\
SCFA-FFA correlations & & & \\
Acetate & $\boldsymbol{r}=\mathbf{0 . 7 7 0}$ & $r=0.067$ & \\
& $\boldsymbol{p}<\mathbf{0 . 0 0 1}$ & $p=0.793$ & \\
Propionate & $\boldsymbol{r}=\mathbf{0 . 7 9 0}$ & $r=0.230$ & \\
Butyrate & $\boldsymbol{p}<\mathbf{0 . 0 0 1}$ & $p=0.359$ & \\
& $\boldsymbol{r}=\mathbf{0 . 7 6 4}$ & $r=-0.066$ & \\
& $\boldsymbol{p}<\mathbf{0 . 0 0 1}$ & $p=0.795$ & \\
& &
\end{tabular}

The differences between fecal SCFA levels found in lupus patients and those in HC were analyzed by Mann-Withney $U$ tests; whereas the correlation analyses were performed by Spearman rank's tests. Variables are summarized as median (interquartile range). Statistical analyses with a p-value below 0.050 are highlighted in bold.
On the other hand, no differences in the PCA scores were registered between SLE and HC groups (PC1: $p=0.169, \mathrm{PC} 2$ : $p=0.378$, and PC3: $p=0.916)$, thus suggesting that SLE patients did not exhibit a different FFA profile compared to $\mathrm{HC}$.

Finally, whether PCA scores could be related to gut microbiota composition was analyzed. Notably, F/B ratio was positively correlated with the PC2 score (Table 8) in HC but not in SLE, thereby suggesting a beneficial effect of gut microbiota composition on the serum FFA pool in healthy individuals. Again, when the phyla were independently studied, Bacteroidetes exhibited a stronger correlation with PC2 score $(r=-0.433, p=0.039)$ than that of Firmicutes $(r=0.411, p=0.052)$ in HC individuals. Thus, gut microbiota seems to quantitatively and qualitatively impact the FFA serum pool.

\section{FFA and Serum Biomarkers in SLE Patients}

Since some associations between gut microbiota and specific FFA PCA scores were observed, we aimed to evaluate whether these 

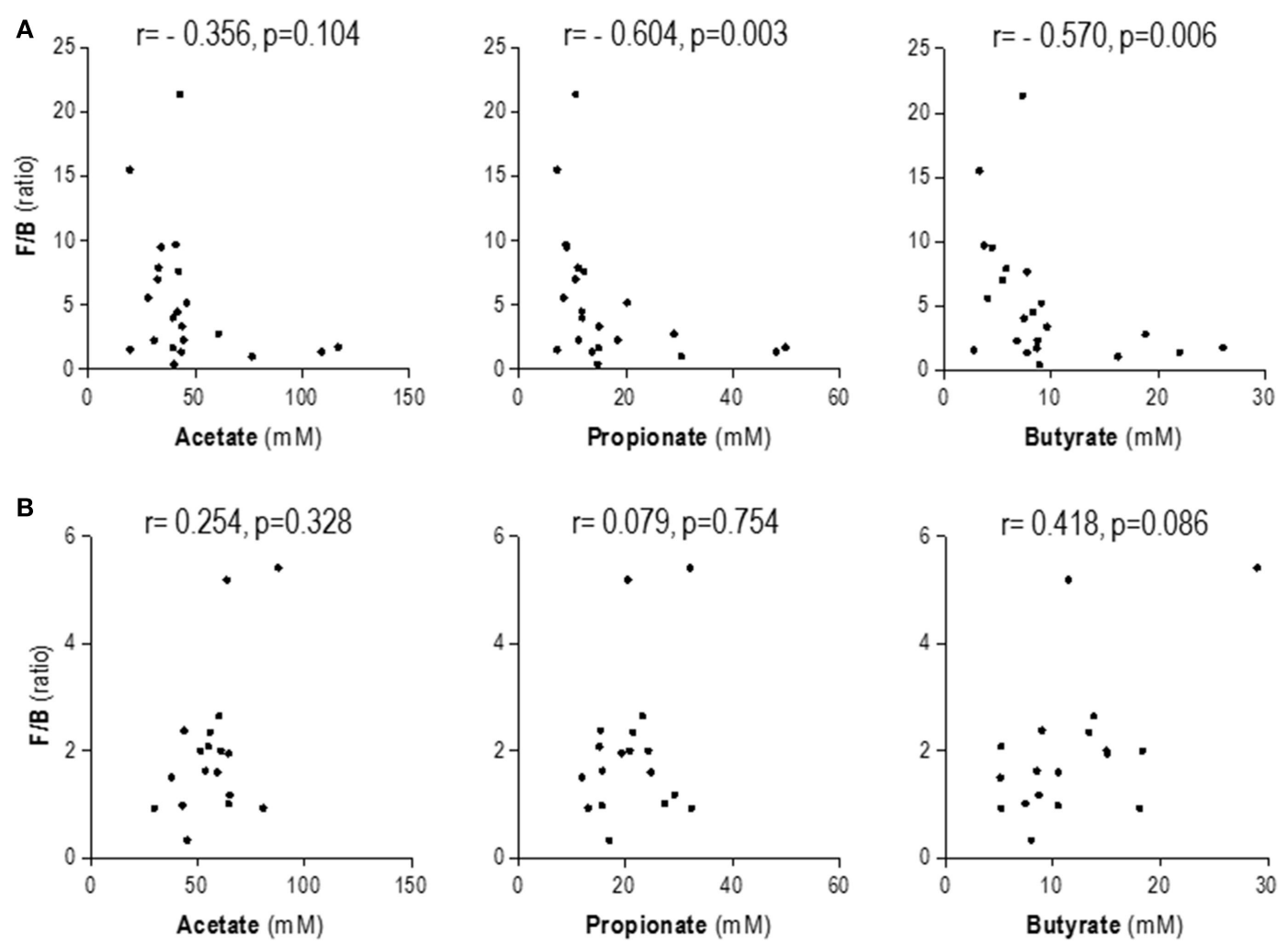

FIGURE 3 | Association between fecal short-chain fatty acids (SCFA) levels and Firmicutes to Bacteroidetes ratio. Correlation analyses by Spearman's ranks tests were performed in order to analyze the association between fecal SCFA levels and those of serum-free fatty acids in healthy controls (A) and systemic lupus erythematosus patients (B).

TABLE 7 | Specific free fatty acids (FFA) in healthy controls (HC) and systemic lupus erythematosus (SLE) patients.

\begin{tabular}{|c|c|c|c|}
\hline FFA $(\mu \mathrm{g} / \mathrm{ml})$ & $\mathrm{HC}$ & SLE & $p$ \\
\hline Palmitic (16:0) & 32.76 (15.59) & 30.35 (8.37) & 0.982 \\
\hline Stearic (18:0) & $28.51(12.95)$ & $29.12(6.19)$ & 0.873 \\
\hline 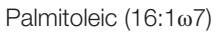 & $1.93(1.62)$ & $2.67(0.99)$ & 0.351 \\
\hline 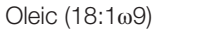 & $27.76(20.61)$ & $34.39(17.77)$ & 0.467 \\
\hline 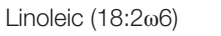 & $6.99(6.47)$ & $10.26(7.35)$ & 0.246 \\
\hline 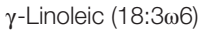 & $0.08(0.08)$ & $0.10(0.06)$ & 0.785 \\
\hline AA $(20: 4 \omega 6)$ & $1.96(1.68)$ & $2.74(1.74)$ & 0.045 \\
\hline 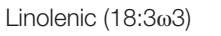 & $0.18(0.17)$ & $0.20(0.16)$ & 0.539 \\
\hline 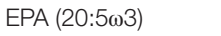 & $0.07(0.15)$ & $0.15(0.17)$ & 0.363 \\
\hline 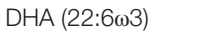 & $1.47(1.46)$ & $1.65(1.76)$ & 0.209 \\
\hline
\end{tabular}

The differences in specific FFA serum levels between control and patients were assessed by Mann-Withney $U$ tests. Variables are summarized as median (interquartile range). Statistical analyses with a p-value below 0.050 are highlighted in bold.

parameters could be related to some relevant serum biomarkers in lupus. To this end, a panel of serum biomarkers of endothelial activation (VEGF, GM-CSF, EGF, IL-8, TNF $\alpha$, MCP-1, IP-10, and leptin) and oxidative stress (MDA) were measured (Table 9).

First, the associations between these biomarkers and the gut microbiota composition were analyzed. Interestingly, the F/B ratio was negatively associated to leptin $(r=-0.369, p=0.006)$ and MCP-1 serum levels $(r=-0.304, p=0.025)$ in the whole group.
On the other hand, divergent associations were noted among FFA PCA scores and these biomarkers in SLE patients. Whereas PC1 was positively correlated with biomarkers of endothelial activation [VEGF $(r=0.444, p=0.044)$, IL-8 $(r=0.522$, $0.015)$, and EGF $(r=0.400, p=0.070)]$, negative associations were observed for PC2 [EGF $(r=-0.425, p=0.055)$, MCP-1 $(r=-0.640, p=0.002)$, IP-10 $(r=-0.397, p=0.075), \mathrm{TNF} \alpha$ $(r=-0.410, p=0.065)$, and MDA $(r=-0.375, p=0.057)]$. Interestingly, negative associations were also found for PC3 [VEGF $(r=-0.534, p=0.013)$ and IL-8 $(r=-0.459, p=0.036)]$. No associations were observed in the HC.

All these findings seem to point to a link between gut microbiota, FFA serum pool, and biomarkers of endothelial activation in lupus, thus emphasizing the systemic effect of the gut microbiota in this condition. Additionally, differences among FFA PCA are in line with their proposed functional diversity.

\section{DISCUSSION}

Over the last decade, several studies have revealed a number of interactions between the gut microbiota and the host in homeostatic conditions. Accordingly, dysbiosis has been consistently related to different pathological situations, from immunemediated to metabolic diseases (27-29). In this sense, we have recently reported the existence of an intestinal dysbiosis in SLE 


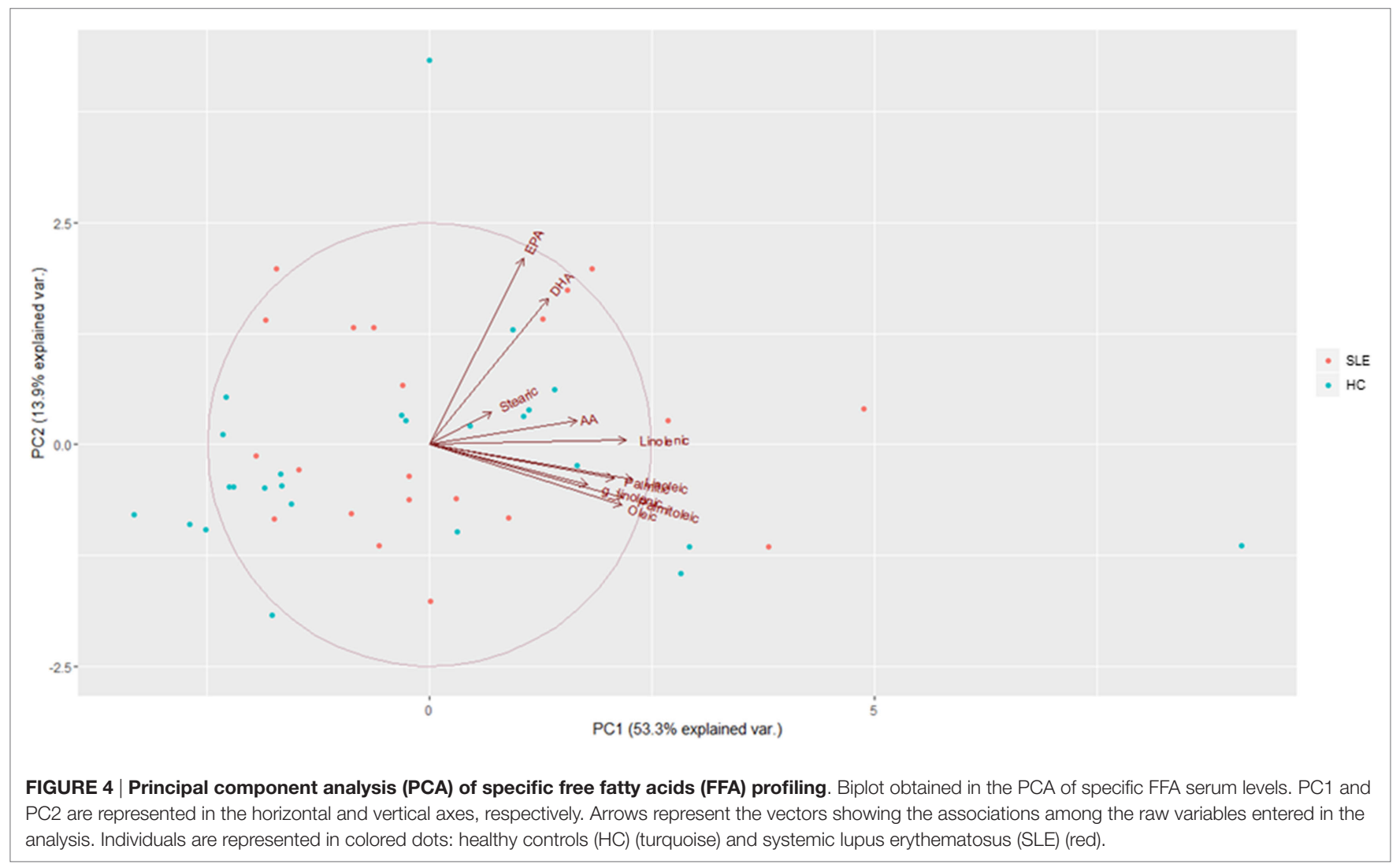

TABLE 8 | Associations between Firmicutes to Bacteroidetes ratio and free fatty acids-principal component analysis scores in healthy controls (HC) and systemic lupus erythematosus (SLE) patients.

\begin{tabular}{llr}
\hline & HC & SLE \\
\hline PC1 & $r=-0.246$ & $r=0.078$ \\
& $p=0.257$ & $p=0.736$ \\
PC2 & $\boldsymbol{r}=\mathbf{0 . 4 3 7}$ & $r=0.110$ \\
& $\boldsymbol{p}=\mathbf{0 . 0 3 7}$ & $p=0.635$ \\
PC3 & $r=-0.075$ & $r=0.089$ \\
& $p=0.734$ & $p=0.700$ \\
\hline
\end{tabular}

Correlations between these parameters were assessed by Pearson correlation tests. Statistical analyses with a p-value below 0.050 are highlighted in bold.

(22). Additional studies from our group allowed us to associate the dysbiotic state with the dysregulated Treg/Th17 responses found in lupus patients (30). In the present report, we go a step further by addressing the study of the potential connections between the intestinal dysbiosis and the metabolic impairment in SLE, focusing on the role of FFA. Thus, gut microbiota may not only be related to disease pathogenesis itself but also to some comorbidities frequently present in lupus patients. Since the origin of such alterations is ill-defined, these new findings allow us to gain some insight into this complex situation and may help to delineate new therapeutic targets. Actually, the experimental modulation of the gut microbiota has yielded promising results in other scenarios (17). In fact, the lupus-like immune over-activation was partially reestablished in vitro by the supplementation with
TABLE 9 | Levels of serum biomarkers analyzed in healthy controls (HC) and systemic lupus erythematosus (SLE) patients.

\begin{tabular}{|c|c|c|c|}
\hline & $\mathrm{HC}$ & SLE & $p$ \\
\hline $\begin{array}{l}\text { Vascular endothelial growth } \\
\text { factor [VEGF] }(\mathrm{pg} / \mathrm{ml})\end{array}$ & $83.38(49.49)$ & $70.73(98.87)$ & 0.326 \\
\hline $\begin{array}{l}\text { Granulocyte monocyte } \\
\text { colonystimulating factor } \\
\text { [GM-CSF] }(\mathrm{pg} / \mathrm{ml})\end{array}$ & $0.35(0.92)$ & $0.35(2.77)$ & 0.424 \\
\hline $\begin{array}{l}\text { Epidermal growth factor [EGF] } \\
(\mathrm{pg} / \mathrm{ml})\end{array}$ & $114.29(81.35)$ & $65.57(78.61)$ & 0.019 \\
\hline Interleukin-8 [lL-8] (pg/ml) & $14.69(22.51)$ & $27.85(21.92)$ & 0.016 \\
\hline $\begin{array}{l}\text { Tumor necrosis factor alpha } \\
{[T N F \alpha](p g / m l)}\end{array}$ & $174.40(309.4)$ & $188.14(292.92)$ & 0.789 \\
\hline $\begin{array}{l}\text { Monocyte chemoattractant } \\
\text { protein-1 [MCP-1] (pg/ml) }\end{array}$ & $444.55(481.45)$ & $616.13(402.07)$ & 0.011 \\
\hline $\begin{array}{l}\text { Interferon gamma-inducible } \\
\text { protein-10 [IP-10] (pg/ml) }\end{array}$ & $98.82(148.81)$ & $167.67(152.76)$ & 0.019 \\
\hline Leptin (ng/ml) & $7.73(7.14)$ & $14.16(20.20)$ & $<0.001$ \\
\hline Malondialdehyde $[\mathrm{MDA}](\mu \mathrm{M})$ & $2.78(0.71)$ & $2.90(0.44)$ & 0.658 \\
\hline
\end{tabular}

The differences in serum levels between control and patients were assessed by Mann-Withney $U$ tests. Variables are summarized as median (interquartile range). Statistical analyses with a p-value below 0.050 are highlighted in bold.

specific bacterial strains (30). Thus, our study warrants future research to assess whether this therapy may be also advisable to counteract the metabolic alterations in SLE patients.

Metabolic disorders, including metabolic syndrome, are common hallmarks of SLE and other related diseases (31-33). 
However, the underlying causes of these traits are not well defined. The findings herein presented suggest that gut microbiota may play a role in this condition. This notion can explain why a wide range of diseases, with striking differences in their clinical presentations, are associated with similar comorbidities (such as metabolic alterations). Thus, it is feasible to think that similar patterns of intestinal dysbiosis may underlie this situation. This idea is supported by the altered $\mathrm{F} / \mathrm{B}$ ratios that were reported in other diseases exhibiting increased serum FFA levels (34-36). The fact that this ratio is a continuum may explain the differences in prevalence and severity of metabolic complications among different conditions. However, it must be remarked that studies on the alterations in the $\mathrm{F} / \mathrm{B}$ ratio have yielded contradictory results in different contexts, such as obesity (37-39). Schwiertz and colleagues have recently published a reduced $\mathrm{F} / \mathrm{B}$ ratio in obese and overweighed individuals in a large study population (40). Similarly, the enrichment of Firmicutes in the intestinal ecosystem has been related to improved lipid absorption and homeostasis in animal models (41). It must be taken into account that differences in sequencing techniques, data analysis, and characteristics of the recruited populations may be an important source of discrepancy in this field. Thus, the associations of the microbiota with metabolic traits in different scenarios must be interpreted with caution, since direct comparison is not always possible. Additional studies focusing on the F/B ratio in nonobese healthy population are warranted.

The association between the F/B ratio and the serum levels of FFA emphasizes the ability of the gut microbiota to promote systemic responses in the host. Moreover, it supports that gut microbiota can modulate the energy metabolism of the host (42). However, the identification of the actual mediators involved and the potential impact of this interaction in pathological conditions is currently lacking in the literature. Our findings in the present work point to the SCFA as potential orchestrators of the cross talk between gut microbiota and the host metabolism. Although these compounds are thought to be of key relevance in the interactions between intestinal microbial populations and the host (16), their link with the lipid metabolism remains controversial. Our results suggest that the SCFA production paralleled the FFA serum levels in healthy individuals. However, this association was absent in the pathological framework of lupus, where an increased SCFA production, together with elevated FFA serum levels, was noted. These results are in line with those from other metabolic conditions [reviewed in Ref. (43)]. Additionally, our analyses revealed that this aberrant SCFA production can be linked to an altered microbial gut composition, as a reduced $\mathrm{F} / \mathrm{B}$ ratio was related to increased SCFA fecal levels. In this scenario, the potential involvement of the propionate deserves a special mention. Propionate is mainly produced by Bacteroidetes species (44), thereby supporting its negative association with the F/B ratio observed. It is important to note that increased propionate fecal levels have been reported in obese individuals exhibiting a decreased F/B ratio (40). Furthermore, exposure of human intestinal organoids to propionate led to an upregulation of genes belonging to lipolytic pathways (45). Moreover, experimental evidence from animal models and ex vivo experiments with human material have identified a mechanistic link between exposure to propionate and increased lipolysis mediated by the increased expression of the enzyme lipoprotein lipase (46-48). Moreover, a homeostatic role for propionate on glucose metabolism and regulation of energy intake has also been proposed. However, which is the actual role for propionate in human diseases required further elucidation. Taken together, our finding may provide a possible explanation for the elevated FFA serum levels in SLE, altered SCFA production, and overrepresentation of Bacteroidetes in the intestinal microbiota playing a pivotal role. The stronger associations of Bacteroidetes alone compared with those of the $\mathrm{F} / \mathrm{B}$ ratio are in line with this point.

Another important result of our study was the association between FFA and some biomarkers of endothelial activation. Because of their nature, FFA are considered as common mediators between immunity, inflammation, and metabolism. Although some authors have previously proposed a role for the gut microbiota in the etiology of metabolic alterations and CVD (49), the actual players are far from being clear. Interestingly, experimental studies revealed a mechanistic link between propionate and leptin expression by human adipose and omental tissues (50), which is in line with our results. Nevertheless, the exact significance of this finding in vivo is not known. Taking into account the effects of FFA on inflammation, oxidative stress, and expression of adhesion molecules (51-53), our results may support a role for FFA as a link between the (altered) gut microbiota, host metabolism, and disease status. It is interesting to note that differences among FFA in their ability to promote endothelial activation in vivo or in vitro have been demonstrated (54-56), which is in line with the associations found in our study. Importantly, these biomarkers are considered as early preclinical indicators of CVD development in the long term (57-60). Taking this into account, these associations may point to a very early role of the altered gut microbiota in the etiology of these complications. This is reinforced by the negative association between the intestinal dysbiosis in lupus and the levels of the protective IgM antibodies against phosphorylcholine (30). These antibodies are known to enhance apoptotic-cell clearance and induce anti-inflammatory pathways, explaining its negative association with markers of subclinical atherosclerosis (61) and CVD development (62) in lupus.

Finally, our approach did not identify a different FFA serum profile in SLE patients in comparison to HC. Although a similar pattern of grouping of FFA species in the PCA was observed in rheumatoid arthritis patients by our group (24), differences among FFA species between patients and controls were not observed in the case of lupus. Interestingly, no differences in plasma FFA profiling were observed in a previous study with lupus patients (63), although slight alterations in the polyunsaturated fatty acids were reported in those with a previous history of CVD. Our results are, at least in part, in line with these findings, although differences in the experimental procedures between both studies are important. It is worthy to note that our group of patients was characterized by a low disease activity, even in the absence of glucocorticoid and immunosuppressive drugs. Thus, a larger study involving SLE patients with a higher degree of disease activity is warranted. However, despite no differences being found in the specific FFA levels, the results from the PCA emphasize the heterogeneity among FFA classes and suggest that FFA, despite being mostly 
unaltered, can develop different roles under different milieu. This hypothesis is in line with current evidence in this field $(64,65)$ and stresses the underestimated significance of FFA as key mediators for the human health.

Due to the lack of direct mechanistic data in our approach, these results pose the question on whether the microbiota composition is responsible of the altered FFA levels or if, alternatively, increased FFA levels may lead to changes in the gut ecology. Based on the literature currently available, several research studies seem to align with the former idea. The fact that no changes in the gut microbiota were related to disease duration (in spite of the wide range of disease duration studied in the present report) is also in line with this idea, probably suggesting that intestinal dysbiosis could be present at the preclinical stages of the disease. Similarly, experiments of fecal transplantation in obese and lean mice also support the causative role of the microbiota in shaping the host metabolism (66). However, due to the role of FFA on inflammation, it is tempting to speculate that these molecules can prompt a shift toward a systemic pro-inflammatory state, which can, in turn, disrupt the intestinal microbiota. Experimental studies with mice have revealed that diet-induced obesity is accompanied by changes in the gut microbiota and damaged intestinal barrier $(67,68)$. Interestingly, a diet with high $\omega 6$ content resulted in intestinal dysbiosis in mice, the inflammatory pathways playing a crucial role (69). Moreover, $\omega 3$ fatty acids seem to counteract the effects of diet- or antibiotics-induced dysbiosis through different mechanisms $(70,71)$. Additionally, an antibacterial effect was observed for some FFA (72). Therefore, it is feasible that a bidirectional cross talk between the gut microbiota and host metabolism is established, with immune circuits participating in this interaction.

In summary, our data indicate that increased serum FFA levels in SLE patients may be associated with changes in the gut ecosystem in the framework of lupus dysbiosis. The association between serum FFA and SCFA supports this notion. Additionally, different associations between FFA species and serum biomarkers of endothelial activation were found, hence not only underscoring the heterogeneity among FFA compounds but also shedding new

\section{REFERENCES}

1. Saegusa J, Irino Y, Yoshida M, Tanaka S, Kogata Y, Kageyama G, et al. GC/ MS-based metabolomics detects metabolic alterations in serum from SLE patients. Clin Exp Rheumatol (2014) 32(1):148.

2. Demir S, Artim-Esen B, Şahinkaya Y, Pehlivan Ö, Alpay-Kanıtez N, Omma A, et al. Metabolic syndrome is not only a risk factor for cardiovascular diseases in systemic lupus erythematosus but is also associated with cumulative organ damage: a cross-sectional analysis of 311 patients. Lupus (2016) 25:177-84. doi:10.1177/0961203315603140

3. Parker B, Bruce IN. The metabolic syndrome in systemic lupus erythematosus. Rheum Dis Clin North Am (2010) 36:81-97, viii. doi:10.1016/j.rdc.2009. 12.004

4. Bartels CM, Buhr KA, Goldberg JW, Bell CL, Visekruna M, Nekkanti S, et al. Mortality and cardiovascular burden of systemic lupus erythematosus in a US population-based cohort. J Rheumatol (2014) 41:680-7. doi:10.3899/ jrheum. 130874

5. Esdaile JM, Abrahamowicz M, Grodzicky T, Li Y, Panaritis C, Berger R, et al. Traditional Framingham risk factors fail to fully account for accelerated atherosclerosis in systemic lupus erythematosus. Arthritis Rheum (2001) 44:2331-7. doi:10.1002/1529-0131(200110)44:10<2331::AID-ART395>3.0.CO;2-I light on the gut-metabolism-CVD axis. Although the reduced sample size and the lack of a mechanistic data are the limitations of our study, to the best of our knowledge this is the first report supporting a connection between gut microbiota, FFA, and biomarkers of endothelial activation. Moreover, we have provided a proof of concept evidence on the involvement of the intestinal dysbiosis in the metabolic alterations in lupus.

\section{AUTHOR CONTRIBUTIONS}

All the authors listed made substantial contributions to the design of the work, analysis, or interpretation of the results obtained; involved in drafting the manuscript, revising it critically for intellectual content, and approving the final version; and agreed to be accountable for all aspects of the work in ensuring that questions related to the accuracy or integrity of any part of the work are appropriately investigated and resolved.

\section{ACKNOWLEDGMENTS}

The authors acknowledge the excellent technical assistance of Ana M. Hernández-Barranco (IPLA) with the analysis of SCFA in fecal samples as well as of the staff of the scientific core facilities from the University of Oviedo (Unidad de Espectrometría y Espectrofotometría, Servicios Científico-Técnicos, Universidad de Oviedo) with the FFA analysis. The authors also show our deepest gratitude to all the study volunteers.

\section{FUNDING}

This work was funded through the grants GRUPIN14-043 "Microbiota Humana, Alimentación y Salud" from the "Plan Regional de Investigación del Principado de Asturias" and cofounded from European Union FEDER funds, AGL201014952 from the Spanish Ministry of Science and Innovation, and PI012/00523 from the "Fondo de Investigaciones Sanitarias, Instituto de Investigación Carlos III." JR-C is supported by a contract from the grant GRUPIN14-043.

6. Bartoloni E, Shoenfeld Y, Gerli R. Inflammatory and autoimmune mechanisms in the induction of atherosclerotic damage in systemic rheumatic diseases: two faces of the same coin. Arthritis Care Res (2011) 63:178-83. doi:10.1002/ acr.20322

7. Symmons DP, Gabriel SE. Epidemiology of CVD in rheumatic disease, with a focus on RA and SLE. Nat Rev Rheumatol (2011) 7:399-408. doi:10.1038/ nrrheum.2011.75

8. López-Pedrera C, Barbarroja N, Jimenez-Gomez Y, Collantes-Estevez E, Aguirre MA, Cuadrado MJ. Oxidative stress in the pathogenesis of atherothrombosis associated with anti-phospholipid syndrome and systemic lupus erythematosus: new therapeutic approaches. Rheumatology (Oxford) (2016) 55(12):2096-108. doi:10.1093/rheumatology/kew054

9. Boden G. Obesity and free fatty acids. Endocrinol Metab Clin North Am (2008) 37:635-46, viii-ix. doi:10.1016/j.ecl.2008.06.007

10. de Jong AJ, Kloppenburg M, Toes RE, Ioan-Facsinay A. Fatty acids, lipid mediators, and T-cell function. Front Immunol (2014) 5:483. doi:10.3389/ fimmu.2014.00483

11. Ormseth MJ, Swift LL, Fazio S, Linton MF, Raggi P, Solus JF, et al. Free fatty acids are associated with metabolic syndrome and insulin resistance but not inflammation in systemic lupus erythematosus. Lupus (2013) 22:26-33. doi:10.1177/0961203312462756 
12. Saito I. Epidemiological evidence of type 2 diabetes mellitus, metabolic syndrome, and cardiovascular disease in Japan. Circ J (2012) 76:1066-73. doi:10.1253/circj.CJ-11-1519

13. Shaw JE, Zimmet PZ, George K, Alberti MM. Metabolic syndrome-do we really need a new definition? Metab Syndr Relat Disord (2005) 3:191-3. doi:10.1089/met.2005.3.191

14. Chang Y, Jung H-S, Yun KE, Cho J, Ahn J, Chung EC, et al. Metabolically healthy obesity is associated with an increased risk of diabetes independently of nonalcoholic fatty liver disease. Obesity (Silver Spring) (2016) 24:1996-2003. doi:10.1002/oby. 21580

15. Cheng FW, Gao X, Mitchell DC, Wood C, Rolston DD, Still CD, et al. Metabolic health status and the obesity paradox in older adults. J Nutr Gerontol Geriatr (2016) 35:161-76. doi:10.1080/21551197.2016.1199004

16. Canfora EE, Jocken JW, Blaak EE. Short-chain fatty acids in control of body weight and insulin sensitivity. Nat Rev Endocrinol (2015) 11:577-91. doi:10.1038/nrendo.2015.128

17. Boulangé CL, Neves AL, Chilloux J, Nicholson JK, Dumas ME. Impact of the gut microbiota on inflammation, obesity, and metabolic disease. Genome Med (2016) 8:42. doi:10.1186/s13073-016-0303-2

18. Brestoff JR, Artis D. Commensal bacteria at the interface of host metabolism and the immune system. Nat Immunol (2013) 14:676-84. doi:10.1038/ ni. 2640

19. Clarke G, Stilling RM, Kennedy PJ, Stanton C, Cryan JF, Dinan TG. Minireview: gut microbiota: the neglected endocrine organ. Mol Endocrinol (2014) 28:1221-38. doi:10.1210/me.2014-1108

20. Sharon G, Garg N, Debelius J, Knight R, Dorrestein PC, Mazmanian SK. Specialized metabolites from the microbiome in health and disease. Cell Metab (2014) 20:719-30. doi:10.1016/j.cmet.2014.10.016

21. Marchesi JR, Adams DH, Fava F, Hermes GD, Hirschfield GM, Hold G, et al. The gut microbiota and host health: a new clinical frontier. Gut (2016) 65:330-9. doi:10.1136/gutjnl-2015-309990

22. Hevia A, Milani C, López P, Cuervo A, Arboleya S, Duranti S, et al. Intestinal dysbiosis associated with systemic lupus erythematosus. mBio (2014) 5:e1548-1514. doi:10.1128/mBio.01548-14.Invited

23. Tan EM, Cohen AS, Fries JF, Masi AT, McShane DJ, Rothfield NF, et al. The 1982 revised criteria for the classification of systemic lupus erythematosus. Arthritis Rheum (1982) 25:1271-7. doi:10.1002/art.1780251101

24. Rodríguez-Carrio J, Alperi-López M, López P, Ballina-García FJ, Suárez A. Non-esterified fatty acids profiling in rheumatoid arthritis: associations with clinical features and Th1 response. PLoS One (2016) 11:e0159573. doi:10.1371/ journal.pone.0159573

25. Cuervo A, Hevia A, López $\mathrm{P}$, Suárez A, Sánchez B, Margolles A, et al. Association of polyphenols from oranges and apples with specific intestinal microorganisms in systemic lupus erythematosus patients. Nutrients (2015) 7:1301-17. doi:10.3390/nu7021301

26. Centro de Enseñanza Superior de Nutrición Humana y Dietética (CESNID). Tablas de composición de alimentos por medidas caseras de consumo habitual en España. Barcelona: McGraw-Hill Publicaciones y Ediciones de la Universidad de Barcelona (2008).

27. Tilg H, Adolph TE. Influence of the human intestinal microbiome on obesity and metabolic dysfunction. Curr Opin Pediatr (2015) 27:496-501. doi:10.1097/ MOP.0000000000000234

28. Peterson CT, Sharma V, Elmén L, Peterson SN. Immune homeostasis, dysbiosis and therapeutic modulation of the gut microbiota. Clin Exp Immunol (2015) 179:363-77. doi:10.1111/cei.12474

29. Honda K, Littman DR. The microbiota in adaptive immune homeostasis and disease. Nature (2016) 535:75-84. doi:10.1038/nature18848

30. López P, de Paz B, Rodríguez-Carrio J, Hevia A, Sánchez B, Margolles A, et al. Th17 responses and natural IgM antibodies are related to gut microbiota composition in systemic lupus erythematosus patients. Sci Rep (2016) 6:24072. doi:10.1038/srep24072

31. Parker B, Ahmad Y, Shelmerdine J, Edlin H, Yates AP, Teh LS, et al. An analysis of the metabolic syndrome phenotype in systemic lupus erythematosus. Lupus (2011) 20:1459-65. doi:10.1177/0961203311416695

32. Sidiropoulos PI, Karvounaris SA, Boumpas DT. Metabolic syndrome in rheumatic diseases: epidemiology, pathophysiology, and clinical implications. Arthritis Res Ther (2008) 10:207. doi:10.1186/ar2397

33. Parker B, Urowitz MB, Gladman DD, Lunt M, Donn R, Bae SC, et al. Impact of early disease factors on metabolic syndrome in systemic lupus erythematosus: data from an international inception cohort. Ann Rheum Dis (2015) 74:1530-6. doi:10.1136/annrheumdis-2013-203933

34. Wong VW-S, Tse C-H, Lam TT-Y, Wong GL-H, Chim AM-L, Chu WC-W, et al. Molecular characterization of the fecal microbiota in patients with nonalcoholic steatohepatitis - a longitudinal study. PLoS One (2013) 8:e62885. doi:10.1371/journal.pone.0062885

35. Zhu L, Baker SS, Gill C, Liu W, Alkhouri R, Baker RD, et al. Characterization of gut microbiomes in nonalcoholic steatohepatitis (NASH) patients: a connection between endogenous alcohol and NASH. Hepatology (2013) 57:601-9. doi:10.1002/hep.26093

36. Larsen N, Vogensen FK, van den Berg FWJ, Nielsen DS, Andreasen AS, Pedersen BK, et al. Gut microbiota in human adults with type 2 diabetes differs from non-diabetic adults. PLoS One (2010) 5:e9085. doi:10.1371/journal. pone.0009085

37. Ley RE, Turnbaugh PJ, Klein S, Gordon JI. Microbial ecology: human gut microbes associated with obesity. Nature (2006) 444:1022-3 doi:10.1038/4441022a

38. Ley RE, Bäckhed F, Turnbaugh P, Lozupone CA, Knight RD, Gordon JI. Obesity alters gut microbial ecology. Proc Natl Acad Sci U S A (2005) 102:11070-5. doi:10.1073/pnas.0504978102

39. Raman M, Ahmed I, Gillevet PM, Probert CS, Ratcliffe NM, Smith S, et al. Fecal microbiome and volatile organic compound metabolome in obese humans with nonalcoholic fatty liver disease. Clin Gastroenterol Hepatol (2013) 11: 868-75.e1-3. doi:10.1016/j.cgh.2013.02.015

40. Schwiertz A, Taras D, Schäfer K, Beijer S, Bos NA, Donus C, et al. Microbiota and SCFA in lean and overweight healthy subjects. Obesity (Silver Spring) (2010) 18:190-5. doi:10.1038/oby.2009.167

41. Semova I, Carten JD, Stombaugh J, Mackey LC, Knight R, Farber SA, et al. Microbiota regulate intestinal absorption and metabolism of fatty acids in the zebrafish. Cell Host Microbe (2012) 12:277-88. doi:10.1016/j.chom.2012.08.003

42. Bäckhed F, Ding H, Wang T, Hooper LV, Koh GY, Nagy A, et al. The gut microbiota as an environmental factor that regulates fat storage. Proc Natl Acad Sci U S A (2004) 101:15718-23. doi:10.1073/pnas.0407076101

43. Ríos-Covián D, Ruas-Madiedo P, Margolles A, Gueimonde M, de Los Reyes-Gavilán CG, Salazar N. Intestinal short chain fatty acids and their link with diet and human health. Front Microbiol (2016) 7:185. doi:10.3389/ fmicb.2016.00185

44. Salonen A, Lahti L, Salojärvi J, Holtrop G, Korpela K, Duncan SH, et al. Impact of diet and individual variation on intestinal microbiota composition and fermentation products in obese men. ISME J (2014) 8:2218-30. doi:10.1038/ ismej.2014.63

45. Lukovac S, Belzer C, Pellis L, Keijser BJ, de Vos WM, Montijn RC, et al. Differential modulation by Akkermansia muciniphila and Faecalibacterium prausnitzii of host peripheral lipid metabolism and histone acetylation in mouse gut organoids. MBio (2014) 5:e01438-14. doi:10.1128/mBio. 01438-14

46. Lee SH, Hossner KL. Coordinate regulation of ovine adipose tissue gene expression by propionate. J Anim Sci (2002) 80:2840-9. doi:10.2527/2002.8 0112840x

47. Merkel M, Eckel RH, Goldberg IJ. Lipoprotein lipase: genetics, lipid uptake, and regulation. J Lipid Res (2002) 43:1997-2006. doi:10.1194/jlr.R200015-JLR200

48. Al-Lahham S, Roelofsen H, Rezaee F, Weening D, Hoek A, Vonk R, et al. Propionic acid affects immune status and metabolism in adipose tissue from overweight subjects. Eur JClin Invest (2012) 42:357-64. doi:10.1111/j.1365-2362.2011.02590.x

49. Harris K, Kassis A, Major G, Chou CJ. Is the gut microbiota a new factor contributing to obesity and its metabolic disorders? J Obes (2012) 2012:879151. doi:10.1155/2012/879151

50. Al-Lahham SH, Roelofsen H, Priebe M, Weening D, Dijkstra M, Hoek A, et al. Regulation of adipokine production in human adipose tissue by propionic acid. Eur J Clin Invest (2010) 40:401-7. doi:10.1111/j.1365-2362.2010.02278.x

51. Mathew M, Tay E, Cusi K. Elevated plasma free fatty acids increase cardiovascular risk by inducing plasma biomarkers of endothelial activation, myeloperoxidase and PAI-1 in healthy subjects. Cardiovasc Diabetol (2010) 9:9. doi:10.1186/1475-2840-9-9

52. Tripathy D, Aljada A, Dandona P. Free fatty acids (FFA) and endothelial dysfunction; role of increased oxidative stress and inflammation. - to: Steinberg et al. (2002) Vascular function, insulin resistance and fatty acids. Diabetologia (2003) 46:300-1. doi:10.1007/s00125-002-1027-y 
53. Steinberg $\mathrm{HO}$, Baron $\mathrm{AD}$. Vascular function, insulin resistance and fatty acids. Diabetologia (2002) 45:623-34. doi:10.1007/s00125-002-0800-2

54. Ishida T, Naoe S, Nakakuki M, Kawano H, Imada K. Eicosapentaenoic acid prevents saturated fatty acid-induced vascular endothelial dysfunction: involvement of long-chain Acyl-CoA synthetase. J Atheroscler Thromb (2015) 22:1172-85. doi:10.5551/jat.28167

55. Newens KJ, Thompson AK, Jackson KG, Williams CM. Endothelial function and insulin sensitivity during acute non-esterified fatty acid elevation: effects of fat composition and gender. Nutr Metab Cardiovasc Dis (2015) 25:575-81. doi:10.1016/j.numecd.2015.03.004

56. Loaiza A, Carretta MD, Taubert A, Hermosilla C, Hidalgo MA, Burgos RA. Differential intracellular calcium influx, nitric oxide production, ICAM- 1 and IL8 expression in primary bovine endothelial cells exposed to nonesterified fatty acids. BMC Vet Res (2016) 12:38. doi:10.1186/s12917-016-0654-3

57. Weber C, Schober A, Zernecke A. Chemokines: key regulators of mononuclear cell recruitment in atherosclerotic vascular disease. Arterioscler Thromb Vasc Biol (2004) 24:1997-2008. doi:10.1161/01.ATV.0000142812. $03840.6 \mathrm{f}$

58. Zuojun $\mathrm{H}$, Lingyu $\mathrm{H}$, Wei $\mathrm{H}$, Henghui $\mathrm{Y}$, Chonggang $\mathrm{Z}$, Jingsong $\mathrm{W}$, et al. Interference of IP-10 expression inhibits vascular smooth muscle cell proliferation and intimal hyperplasia in carotid artery: a new insight in the prevention of restenosis. Cell Biochem Biophys (2012) 62:125-35. doi:10.1007/ s12013-011-9270-9

59. Boring L, Gosling J, Cleary M, Charo IF. Decreased lesion formation in CCR2-/mice reveals a role for chemokines in the initiation of atherosclerosis. Nature (1998) 394:894-7. doi:10.1038/29788

60. Apostolakis S, Vogiatzi K, Amanatidou V, Spandidos DA. Interleukin 8 and cardiovascular disease. Cardiovasc Res (2009) 84:353-60. doi:10.1093/cvr/ crp241

61. Grönwall C, Reynolds H, Kim JK, Buyon J, Goldberg JD, Clancy RM, et al. Relation of carotid plaque with natural IgM antibodies in patients with systemic lupus erythematosus. Clin Immunol (2014) 153:1-7. doi:10.1016/j. clim.2014.03.017

62. Su J, Hua X, Concha H, Svenungsson E, Cederholm A, Frostegård J. Natural antibodies against phosphorylcholine as potential protective factors in SLE. Rheumatology (Oxford) (2008) 47:1144-50. doi:10.1093/rheumatology/ ken 120

63. Aghdassi E, Ma DW, Morrison S, Hillyer LM, Clarke S, Gladman DD, et al. Alterations in circulating fatty acid composition in patients with systemic lupus erythematosus: a pilot study. JPEN J Parenter Enteral Nutr (2011) 35:198-208. doi:10.1177/0148607110386378

64. Perreault M, Roke K, Badawi A, Nielsen DE, Abdelmagid SA, El-Sohemy A, et al. Plasma levels of 14:0, 16:0, 16:1n-7, and 20:3n-6 are positively associated, but 18:0 and 18:2n-6 are inversely associated with markers of inflammation in young healthy adults. Lipids (2014) 49:255-63. doi:10.1007/ s11745-013-3874-3

65. Alcock J, Lin HC. Fatty acids from diet and microbiota regulate energy metabolism. F1000Research (2015) 4:738. doi:10.12688/f1000research.6078.1

66. Ridaura VK, Faith JJ, Rey FE, Cheng J, Duncan AE, Kau AL, et al. Gut microbiota from twins discordant for obesity modulate metabolism in mice. Science (2013) 341:1241214. doi:10.1126/science.1241214

67. Kless C, Müller VM, Schüppel VL, Lichtenegger M, Rychlik M, Daniel H, et al. Diet-induced obesity causes metabolic impairment independent of alterations in gut barrier integrity. Mol Nutr Food Res (2015) 59:968-78. doi:10.1002/ mnfr.201400840

68. Cani PD, Bibiloni R, Knauf C, Waget A, Neyrinck AM, Delzenne NM, et al. Changes in gut microbiota control metabolic endotoxemia-induced inflammation in high-fat diet-induced obesity and diabetes in mice. Diabetes (2008) 57:1470-81. doi:10.2337/db07-1403

69. Ghosh S, Molcan E, DeCoffe D, Dai C, Gibson DL. Diets rich in n-6 PUFA induce intestinal microbial dysbiosis in aged mice. Br J Nutr (2013) 110:515-23. doi:10.1017/S0007114512005326

70. Kaliannan K, Wang B, Li XY, Bhan AK, Kang JX. Omega-3 fatty acids prevent early-life antibiotic exposure-induced gut microbiota dysbiosis and later-life obesity. Int J Obes (Lond) (2016) 40:1039-42. doi:10.1038/ijo. 2016.27

71. Ghosh S, DeCoffe D, Brown K, Rajendiran E, Estaki M, Dai C, et al. Fish oil attenuates omega- 6 polyunsaturated fatty acid-induced dysbiosis and infectious colitis but impairs LPS dephosphorylation activity causing sepsis. PLoS One (2013) 8:e55468. doi:10.1371/journal.pone.0055468

72. Shin SY, Bajpai VK, Kim HR, Kang SC. Antibacterial activity of bioconverted eicosapentaenoic (EPA) and docosahexaenoic acid (DHA) against foodborne pathogenic bacteria. Int J Food Microbiol (2007) 113:233-6. doi:10.1016/ j.ijfoodmicro.2006.05.020

Conflict of Interest Statement: The authors declared no financial conflicts of interest. The funders have no role in study design, data analysis, or decision to publish.

Copyright (๑) 2017 Rodríguez-Carrio, López, Sánchez, González, Gueimonde, Margolles, de los Reyes-Gavilán and Suárez. This is an open-access article distributed under the terms of the Creative Commons Attribution License (CC BY). The use, distribution or reproduction in other forums is permitted, provided the original author(s) or licensor are credited and that the original publication in this journal is cited, in accordance with accepted academic practice. No use, distribution or reproduction is permitted which does not comply with these terms. 